\title{
Monocyte to Leukocyte Ratio
}

National Cancer Institute

\section{Source}

National Cancer Institute. Monocyte to Leukocyte Ratio. NCI Thesaurus. Code C64824.

The determination of the ratio of monocytes compared to leukocytes in a blood sample.

The measurement may be expressed as a ratio or percentage. 